\title{
Group Diversity and Salience: A Natural Experiment from a Television Game Show
}

\author{
Gabriella A. Bucci and Rafael Tenorio *, \\ DePaul University
}

\begin{abstract}
$\underline{\text { Abstract }}$
We take advantage of a naturally occurring experiment in a television game show to study the impact of group characteristics on their ability to select salient solutions in a matching game. The Family Feud features families seeking to earn prizes by matching the results of public opinion surveys on various subjects. Our main result is that, controlling for task difficulty, families that are more diverse, as measured by both the intra-family generational gap and the relatedness of their members, are more successful at matching wider ranges of survey responses. This highlights the importance of member diversity in expanding information and decision frames of reference within a group.
\end{abstract}

JEL Codes: C70, C90, D70

Key words: group decisions; diversity; salience; natural experiments.

* Corresponding author: Rafael Tenorio, Department of Economics, DePaul University, 1 E. Jackson Blvd. - Suite 6200, Chicago, IL 60604; rtenorio@condor.depaul.edu (email); (312) 362-8309 (telephone); (312) 362-5452 (fax).

$\S$ We thank an anonymous referee for highly valuable comments. We also thank Scott Baier, Robert Battalio, and various seminar participants for useful feedback. DePaul University’s Kellstadt Graduate School of Business provided generous financial support. 


\section{Introduction}

The impact of member characteristics on group decisions and performance has been the subject of many studies, both theoretical and empirical. One of the issues in this literature is the importance of group diversity or heterogeneity on decision processes and outcomes. The organizational behavior and team composition literature conclude that there are tradeoffs between heterogeneous and homogeneous teams in terms of their task completion performance. Heterogeneous teams have access to more varied information sources for decision-making, but homogeneous teams are more harmonious and therefore expend less effort on coordination and implementation. As a result of this tradeoff, some empirical studies show that homogeneous groups outperform heterogeneous groups (O’Reilly and Flatt 1989, Ancona and Caldwell 1992), while others show that heterogeneous groups outperform homogeneous groups, especially if there is much uncertainty and the stakes are high (Mello and Ruckes, 2006, Gruenfeld et. al. 1996, Hamilton et. al. 2003). What is clear from this literature is that both the nature of the task and the team's composition determine the outcome of the task and the overall team payoff (Prat 2002, Adamowicz et. al. 2005).

The literature identifies two main mechanisms by which member diversity affects a team's effectiveness in implementing strategies and completing a task (Hinsz et. al., 1997). The first is a pure information effect, with more diverse teams being more likely to hold more pieces of unique or unshared information. ${ }^{1}$ The second mechanism works through the heterogeneity of ideas likely to be present in diverse groups. This

\footnotetext{
${ }^{1}$ The term unshared information is used to refer to information that is uniquely held by one or more, but not all, members of the group. See Fleming and Kaiwi, 2002.
} 
heterogeneity increases the availability of frames of reference from which group members approach the task at hand. Although both mechanisms typically affect group performance (e.g., frames of reference are often used to process information), the specific impact of diversity on performance may vary in different contexts (Hamilton, 2004). This is because, independently of group characteristics, the structure of the task ultimately determines the set of strategies that can be applied towards task completion. For example, if developing innovations is the goal, a heterogeneous team will generally outperform a homogeneous one. Alternatively, if agreeing, getting along, and fitting well together are primordial to fulfilling the task, a more homogeneous team will likely be more successful (Prat, 2002).

This paper contributes to this literature by analyzing how member characteristics affect a group's ability to perform a task in a naturally occurring experiment that is part of a television game show. The long running U.S. game show The Family Feud features groups (families) seeking to earn monetary prizes by matching the results of public opinion surveys in a variety of categories, such as show business, work, politics, history, etc. Because the playing families differ in several dimensions, such as gender and age composition, ethnicity, and experience, we are able to analyze the impact of all of these factors in their success at playing the game. Most importantly, because the groups are families with varying compositions, we are also able to study the impact of a yet unexplored dimension of heterogeneity; that of a group's relatedness or genetic-based diversity. Our results show that both the generational gap within the family, as well as how closely related the family is in a genetic sense, are important determinants of how well the family does in this game. More distantly related families and families 
comprising more generations do better than more closely related and close in age families. We attribute this to more diverse groups having expanded sets of information and frames of reference to use in playing this matching game.

Our analysis also makes a contribution to the empirical understanding of the determinants of salience. The concept of salience was originally introduced by Schelling (1960), and later elaborated by many authors in the fields of game and decision theory. Lewis (1969) defines a salient solution as one that "stands out from the rest by its uniqueness in some conspicuous respect.” Salience is important because it is the basis for the conceptualization of a focal point, which is a variety of salient solution that is used to solve coordination problems in games with multiple equilibria. ${ }^{2,3}$ Because of the open-ended way salience is defined, it is not clear why some solutions are more salient than others. In fact, Schelling argues that salience and focal points may depend on "analogy, precedent, time, place, and who the people are." In the game we analyze, the survey subjects have no intrinsic motivation to coordinate with anyone, so their responses reflect what is prominent or salient to them. Thus, the contesting families play a matching game in which success depends on their ability to figure out what is salient to the survey subjects. Since we find that more diverse or heterogeneous families are more successful at matching a wider variety of survey responses, our results lend support to Schelling's idea that "who the people are" is an important determinant of their ability to select salient solutions in situations involving matching or coordination.

\footnotetext{
${ }^{2}$ See surveys by Jansen (1998) and Aydinonat (2001).

${ }^{3}$ Although the game we analyze is not a coordination game per se, the salience criterion applies to both individual decision problems as well as coordination games.
} 


\section{Matching Games and Salience}

The type of game we will study in this paper is a variation of a matching or mentioning game (Bacharach 1993, Sugden 1995). In our game, we have two types of players, strategic and non-strategic. Members of a large group of non-strategic players simultaneously and independently move first, and must mention an element that is part of a set or category, e.g., mention a male singer, or mention a hotel chain. Each nonstrategic player receives the same payoff no matter what his choice is. Once these choices have been rendered, a strategic player, drawn from the same population as the non-strategic players, moves and tries to match a response given by the non-strategic players. The strategic player's payoff is proportional to how frequently her response was given by the non-strategic players.

Suppose that the set from which both types of players draw their choices is $\boldsymbol{W}$, where $\boldsymbol{W}$ has n elements. Each player describes these elements to herself using a process termed labeling. Non-strategic players do not have a rational choice to make. They render a choice because a choice has to be rendered, but there is no endogenous reason to choose in one way or another. Presumably, they will select an element that they consider prominent in virtue of some exogenous factor. We call this a primary salient choice, or choice with a primary salient label. The strategic player, on the other hand, will render a choice based on what she believes the majority of the non-strategic players selected. We call this a secondary salient choice, or a choice with a secondary salient label. ${ }^{4}$

It must be the case that each player attaches labels to the elements of $\boldsymbol{W}$ based on the frequency with which they have been exposed to them, or the frequency with which

\footnotetext{
${ }^{4}$ This terminology was coined by Mehta et. al., 1995.
} 
they occur in their sample. We define a player's sample as the collection of items or objects she has been exposed to through reading, conversation, viewing, or any kind of communication or transmission. Since non-strategic and strategic players come from the same population, there will be a great deal of sample overlap among them, and thus secondary salient choices will closely track the distribution of primary salient choices. However, a player's sample is also determined by individual or idiosyncratic characteristics (e.g., age, gender, ethnicity), and also by characteristics of the environment surrounding her (e.g., nationality, place of residence, household type, education). Thus, secondary salient choices will not always perfectly correlate with primary salient choices. For instance, in the game "name a male singer," someone who was born in the 1930's may be more inclined to choose Frank Sinatra, while someone born in the 1990's may be more inclined to choose Eminem. But someone with a birth place outside the U.S. may choose someone different altogether like Maurice Chevalier or Julio Iglesias, and someone who is an opera aficionado may choose Luciano Pavarotti. How often the singer has appeared in the player's sample will determine the label she attaches to each strategy. The more common the players' samples are, the higher the correlation of the player's labels for the various strategies. Players with similar samples will tend to rank their choices in a similar, though not necessarily identical, way.

Some studies have shown that for most categories the distribution of the frequency of mentioning is highly skewed, with a few elements mentioned very frequently and many elements mentioned very rarely. ${ }^{5,6}$ This suggests that the most

\footnotetext{
${ }^{5}$ As cited by Sugden (1995), Ijiri and Simon (1977) show that few authors publish many papers in Econometrica over a 20 year period, while many authors published one or two
} 
common elements appear very frequently in most people’s samples, but there must be some people for whom the less common elements figure prominently in their sample (just like Pavarotti did for the opera lover). In terms of performance in a matching game, this implies that the most frequently mentioned elements are easy to match for most players, while the less frequently mentioned choices are difficult to match for most players, but may be easy to match for some players (e.g., there is a chance, albeit small, that the strategic player has had exposure to opera music and will select Pavarotti). We will later show that this skewness of the response distributions plays a crucial role in players' ability to match public opinion survey answers.

\section{The Family Feud}

We use information from the U.S. television game show The Family Feud to study an extension of the basic matching game outlined in the previous section. This popular show was first launched in 1976 and continues to air today. It was the number one daytime television show from 1977 through 1979 and remained the number one syndicated show until 1984. It originally aired once per week and moved to five days per week in 1980. The show has undergone several changes in host, beginning with Richard Dawson in 1976, Ray Combs in 1988, Louie Anderson in 1999, Richard Karn in 2002,

papers. In the current paper, the word "group” appears 46 times, the word "salience” appears 30 times, while there are many words that only appear one or two times.

${ }^{6}$ Mathematically, this distribution can be approximated by a Zipfian distribution. See for instance Egghe (2005). 
and John O’Hurley since 2006. There are also many versions airing in a number of countries worldwide. ${ }^{7}$

The format of the Family Feud games has changed very little since the show's original airing. All of the games are based on trying to match responses to surveys of one hundred randomly selected individuals. The surveys are collected prior to the show, and feud players know the surveys are taken from a random sample of 100 respondents. In each show two teams of five family members compete against each other. Each team arranges its members in a predetermined order of their choice, and this order must be maintained through all of the games played on each show.

In the first segment of each show, which is called the feud, the first members from each team face-off in a preliminary buzzer round. In this round, a survey category is presented and the player who gives the response with the higher number of entries in the survey wins the round. The winning player's family then controls play of the feud. In the "old" shows (1976-1985), the buzzer winner's team had the option of playing the feud game or passing (i.e., letting the other team play). In the "new" shows (1988-1995), the buzzer winner's team must play. Recent versions of the game use the old show rule.

Once the playing family has been determined, the feud starts. In this game, each member of the playing family sequentially gives her own answer to try to match the remaining responses to the survey question presented in the buzzer round. Family members may not communicate with each other, but they do hear all the answers given. Immediately after each player gives an answer, all players (on both teams) learn whether the answer given matches a survey response or not and, in the case of a match, the

\footnotetext{
${ }^{7}$ See http://en.wikipedia.org/wiki/Family_Feud for a brief history of the show.
} 
number of survey entries associated with the matched response. Each team member must give a response that is different from those already given. Families earn points for each matching answer they give. The points equal the number of survey respondents who had given the same answer. ${ }^{8}$ The playing family automatically wins the feud if they match all survey answers. They are allowed up to three wrong (non-matching) answers, after which the opposing family has the opportunity to steal the points the playing family has amassed in the feud. To steal the points and win the feud, the opposing family must provide one as yet unmatched response to the survey. ${ }^{9}$ Should the opposing family fail to steal, the playing family wins the feud and keeps the points they accumulated.

After a feud is over, families go back to the buzzer round, and a brand new feud, with a new survey category, starts. During a typical airing of the show three to five feuds are played, until one of the families accumulates 300 points. At that point, they are declared the winners of the game and proceed to the fast money segment, which involves two members of the winning family playing for additional prizes.

The following is an example of an actual feud game. Family A faces Family B in this segment. The members -in order- for Family A are A1, A2, A3, A4, and A5; while the members for Family B are B1, B2, B3, B4, and B5 (this order is determined by the families themselves). The survey question is "Name an animal that lives on both land and water." Family members A1 and B1 face off in the buzzer round. A1 pushes the buzzer button first, and her response is "Frog," which was the third highest on the survey

\footnotetext{
${ }^{8}$ Though in later feuds of the same show, these points may double or triple.

${ }^{9}$ The opposing family is allowed to make this decision as a group, but they only get one chance.
} 
with 19 responses. B1's response is "Seal," which was the fourth highest on the survey with 10 responses. Thus family A controls the game and, in this case, they choose to play. The subsequent responses from the members of family A are:

A2: $\quad$ Duck - 0 responses (first incorrect answer)

A3: $\quad$ Snake - 0 responses (second incorrect answer)

A4: $\quad$ Turtle - 25 responses (highest in the survey)

A5: Crocodile - 22 responses (second highest in the survey)

A1: Tadpole - 0 responses (third incorrect answer)

At this point, because Family A has accumulated three incorrect answers, Family B gets the chance to steal A's points. As a group, Family B's response is "Beaver," which was fifth highest in the survey with 9 responses. Thus family B wins and scores 76 points (the stealing answer points do not count towards the total).

From the preceding description, the feud game is a team version of the matching game we introduced in section II. As in the basic matching game, each strategic player seeks to match responses that are primary salient to a group of non-strategic players randomly drawn from the population. Survey answers are primary salient because they are given as non-strategic individual choices or opinions, with no intention of coordinating. What makes the game richer than its basic version is that the strategic players are members of a team, and, in succession, they will try to match not just the highest ranked or overall primary salient response, but also less popular responses or responses that are primary salient to smaller subsets of survey respondents. As discussed before, most mentioning processes of this type display a highly skewed response 
distribution, which makes the matching increasingly difficult when it comes to lowerranked survey entries.

Because contestants seek to match answers that are primary salient to survey respondents, the contestants will do best by using secondary salience as their decision criterion. However, the high correlation between primary and secondary salient labels, especially for the most popular responses implies that contestants may also do well using primary salience when trying to match those popular answers. But as the most popular responses are eliminated, each player will have to use secondary salience to maximize her chance of making successful matches.

Possible intra and inter-family games do not alter our claim that secondary salience is likely to be the decision rule used by contestants. Consider the intra-family game in which one member of a family has an incentive not to pick the most popular response because it is the only response a subsequent family member will be able to give. She will be trying to match the second, third, fourth, or fifth most popular response in the survey which is consistent with secondary salience. There may also be an inter-family game of trying to block the opponent's ability to steal. This motivation is still consistent with seeking secondary salience because the best way to block the opposing team is to match as many survey responses as possible. Thus the game of "block the other family from stealing" is equivalent to the game of "match the survey responses." In sum, although we cannot entirely rule out intra or inter family games, it is reasonable to conclude that individual players will behave in a way that is consistent with seeking secondary salience, especially as they try to match the less popular survey responses. 
Empirically, our goal is to analyze the determinants of the playing family's success in playing this matching game. Although our metric is the team's success, this success ultimately depends on the success of each individual player at matching the yet unmatched responses in the survey. At the same time, the success of each individual player will likely be affected by the success or failure of previous team members. Based on the framework we outlined in the previous section, and consistent with the literature on group performance, we expect that both team composition and task structure will affect a family's overall success at matching the survey responses. Team composition is measured by characteristics that capture the backgrounds and demographics of the playing family members. These are the factors that will affect the members' labeling of strategies and thus their ability to match survey responses. Task structure is measured by variables designed to capture the skewness of the distribution of survey responses. These variables measure the degree of difficulty of the survey.

\section{Data}

We use data from 524 feuds recorded on VHS tapes. Of these, 200 are from the old shows (Richard Dawson period), where the buzzer winner could choose to play or pass; and 324 are from the new shows (Ray Combs period), where the buzzer winner automatically played the feud. ${ }^{10}$

As with most game show-based natural experiments, selectivity of participants may be an issue. In the case of the Family Feud, there are two potential selectivity

${ }^{10}$ Shows were recorded off The Game Show Network over several months in 1997-1998. The information from each feud was manually written into a template, and then entered into a data base and doubled-checked for consistency over these two stages. 
problems. First, interested participants must call a number in the Southern California area to express their interest in playing the game. Second, all those who call are initially screened by the producers to make sure that they meet certain basic requirements (minimum age, type of family relationship), and, based on that screen, they must audition for the show in person. Thus, the population of game participants is drawn from individuals who are interested in the show (e.g., Family Feud fans), and presumably meet certain personality profiles (e.g., entertaining or outgoing).

We consider two main sets of variables in assessing matching performance; family characteristics and survey characteristics:

\section{i. Family Characteristics}

Because of the various categories and attributes of the public opinion surveys in the feud, the demographics of the playing family will be important in determining how well the family does in matching survey responses and thus scoring points. Each family consists of individuals of various ages, potentially different genders, as well as some degree of common backgrounds and experiences. As stressed before, gender and age of a contestant are potentially important variables because they affect individual samples. In the case of age, both the average level and the dispersion of this variable within the family may play a role. ${ }^{11}$ The average measures overall or cumulative experience or knowledge, while the dispersion measures the impact of generational heterogeneity. Simple measures of age dispersion, such as the variance and standard deviation of the

\footnotetext{
${ }^{11}$ The host sometimes asked contestants what their age was, and all of these were registered in our data base. If the true age was missing, we estimated it based on the participant observable attributes.
} 
family members' ages are potentially sensitive to outliers, and may not accurately represent the effect of generational gaps within the group. Instead, we construct a generational gap index by taking each of the bilateral age gaps across family members, adding them up, and then normalizing the sum by twenty years, an approximate length of a (cultural) generation. ${ }^{12}$

In addition to age and gender, close-knit families are more likely to display higher correlation of the members' salient labels. For instance, a family comprised of both parents and three offspring will have shared a great deal of common experiences (e.g., lived in the same area, traveled to the same places, watched common TV shows, socialized with the same people, etc.). Thus, their samples are more overlapping and their information and salient labels more correlated. On the other hand, a family comprised of a parent, an offspring, an in-law, a cousin, and a nephew, will likely have been exposed to more diverse experiences and show more dissimilar backgrounds and samples. If by virtue of their relationship individual family members have common samples, they are more likely to come up with similar matches to survey questions, which

${ }^{12}$ A cultural generation is usually defined as a group of individuals that "receive a distinctive imprint from the social and political events of its youth" (Schuman and Scott, 1989). This notion is different from that of a biological or familial generation, which refers to the average length of time between a mother's first offspring and her daughter's first offspring. The latest figure for the length of a familial generation in the U.S. -as of 2006- was exactly 25 years (Martin et. al., 2009). Although there is no consensus on the length of a cultural generation, it is generally agreed that it is five to ten years shorter than a familial generation (Schuman and Scott, 1989). 
make each individual, and thus the family as a group, less proficient at matching a variety of responses. To measure this degree of commonality or homogeneity among the members of playing families, we borrow from the population genetics literature, and calculate a family relatedness index based on Sewall Wright's coefficient of relatedness or relationship. ${ }^{13}$ The idea is that the more closely related the family members are, the more likely they will have shared common backgrounds and experiences, thus the more correlated their salient labels are. Table 1 shows the relatedness coefficient that corresponds to each possible relationship within a family.

\section{[Table 1 about here]}

As we see in Table 1, there are ten possible relationships among the five players, which generate ten bilateral relatedness coefficients. We then add all of these bilateral relatedness coefficients to generate a Wright Index of family relatedness. Assuming there are no identical twins -there were none in our sample- the maximum value of this index is 5. We also calculate an Extended Wright Index for each family that assigns a weight of 0.5 to spousal relationships. We do this because, although spouses are not genetically related, they may exhibit a degree of sample commonality because of shared experiences during their adult life. This weight may also capture possible assortative matching. ${ }^{14}$

Table 2 shows the characteristics of the playing families. In addition to the variables we just described, we also include an experience variable to capture possible

\footnotetext{
${ }^{13}$ In simple terms, this coefficient is defined as the fraction of genes shared by two individuals (Wright, 1922).

14 The assortative matching hypothesis states that in a marriage market, competition for spouses results in sorting of partners by characteristics. See Becker (1973).
} 
effects of having played the game before. This variable shows that nearly half of the playing families had already played and won earlier games. Gender-wise, slightly more than half of the contestants in our sample were females, but there was variation in the male to female ratio across families. The average age in our typical playing family was about 35 years old, and the head of the household (first person in the lineup) was about 6 years older than the family average. In terms of ethnicity, more than three-fourths of the playing families were Caucasian, with Blacks being the only minority exceeding $10 \%$ of the total sample. In terms of relatedness, note that the mean Wright and Extended Wright indexes are similar across the entire sample, but they can actually be quite different for any given family depending on the number of spousal relationships. The correlation coefficient between the total generation gap and the Wright index is 0.1915 , while that between total generation gap and the extended Wright Index is 0.2148 . This suggests that families composed of individuals of more vastly different ages were also more likely to be closer blood relatives. The larger figure for the extended Wright index is not surprising because married persons are typically close in age.

\section{[Table 2 about here]}

\section{ii. Survey Characteristics}

The characteristics of a survey also affect a family's success in matching the responses. Some categories may be inherently easier to match than others. Similarly, some questions may have been intentionally designed to be easier to match. ${ }^{15}$ The distribution of survey categories is shown in Table 3. Vocabulary (e.g., "mention a word

\footnotetext{
${ }^{15}$ If the first or second feud took long to complete, subsequent feuds featured easier surveys to expedite the completion of the game.
} 
or phrase that means 'to tell on'”) was the most popular category accounting for more than 26 percent of survey categories. Other popular categories involved Show Business (e.g., "name a sitcom character”), Social (e.g., "name a clothing item that people wear to formal engagements”), and Domestic (e.g., “name a kitchen utensil”).

\section{[Table 3 about here]}

We use three alternative measures to capture the degree of difficulty of a survey. First, we determine the strength of the overall primary salient answer in the survey. We define a survey with very strong primary salience as one in which more than 50 percent of survey respondents gave the top ranked answer. A somewhat weaker definition is a survey with strong primary salience, in which more than 40 percent of respondents gave the top ranked answer. ${ }^{16}$ Second, we consider the number of answers in a survey as an indicator of its degree of difficulty. The more answers there are, the more difficult it will be to match all of them, especially the ones with fewer respondents. Finally, we also consider a Coordination Index, as in Mehta et. al. (1994):

$$
C=\sum_{i=1}^{k}\left(\mathrm{~m}_{\mathrm{i}} / \mathrm{N}\right)\left[\left(\mathrm{m}_{\mathrm{i}}-1\right) /(\mathrm{N}-1)\right]
$$

where:

$\mathrm{k}$ : Number of recorded answers in the survey,

$\mathrm{m}_{\mathrm{i}}$ : Number of individuals giving answer $\mathrm{i}$, and

$\mathrm{N}$ : Number of survey respondents

This index measures the probability that two individuals chosen at random give the same answer to a survey question. It equals 0 if everyone responds differently and 1

${ }^{16}$ Moderate changes in these definitions do not affect the results. 
if everyone gives the same answer. The coordination index is an indicator of the difficulty that players face when trying to match answers in a given survey.

Table 4 summarizes these survey difficulty characteristics. The overall primary salient answer is very strong in just over 50 percent of surveys and strong in 71 percent of surveys. The mean coordination index in the sample indicates that, overall, there is a $34.3 \%$ probability that two randomly selected people will give the same answer to a typical survey question. However, as shown in table 4, this probability can be as low as $20 \%$ for first feud surveys, and as high as $54 \%$ in fourth feud surveys, confirming that, by design, it is easier to score more points in later feuds. The main reason for this is that as the show progresses families need to score more points more quickly for the winner to be able to amass the required 300 points to win. This is also why the number of points may either double or triple in later feuds. As we see in this table, only $40 \%$ of first feuds had a very strong overall primary salient answer, but this percentage more than doubled in fourth feuds. In fact, the strength of the overall primary salient response increases with feud number, and the total number of responses declines in later surveys.

\section{[Table 4 about here]}

\section{Matching and Feud Performance}

Table 5 presents data on the number of responses in a survey and on the frequency of misses by feud contestants per ranked answer. Whereas virtually all the surveys in our data set contained a first, second, and third response, only a little more than $10 \%$ of the surveys contained seven or more responses. On average, the overall primary salient answer was given by nearly 51 out of 100 survey respondents in our sample, and contestants failed to match this answer in fewer than $2 \%$ of the feuds. The 
second and third-ranked survey answers had averages of 19 and 10 respondents, and the contestant miss rates were about $10 \%$ and $21 \%$, respectively. ${ }^{17}$ However, once we get to survey responses ranked fourth and lower, the percent of missed responses becomes large very quickly. In fact, the overall miss rate for survey entries ranked between 4 and 9 was $42.7 \%$, with responses ranked sixth and lower missed more than $50 \%$ of the time. Thus, as predicted, survey responses are highly skewed, which in turn makes the lower-ranked responses increasingly difficult to match for any individual feud contestant.

\section{[Table 5 about here]}

Table 6 summarizes the characteristics of the feuds in our sample. The playing family usually wins the feud and almost always matches the overall primary salient response. In fact, when the buzzer winning family had the option of either playing or passing, they chose to play $80 \%$ of the time (160 out of 200 instances), and they were successful $78 \%$ of those times. This indicates that, when given the choice, it is a good idea to choose to play. There were 194 successful steals in our sample which represents 47 percent of the 413 attempted steals. All of the games in our sample had at least three feuds. Fewer than half the games had four feuds. No games had more than four feuds.

\section{[Table 6 about here]}

\section{(i) Playing Family Performance}

\footnotetext{
${ }^{17}$ The relatively high success rate in matching the second-highest response is consistent with experiments by Heath et. al. (2006). They find that individuals who intentionally seek to diverge from majority choices are successful in coordinating on "second most salient” answers in experiments similar to those of Mehta et. al. (1994).
} 
Tables $7 \mathrm{a}$ and $7 \mathrm{~b}$ show the results of ordinary least squares regressions where the dependent variable is the number of points scored by a playing family, and the

independent variables are the family (team attributes) and survey (task structure) characteristics. By construction, the coordination index is highly negatively correlated with the number of possible answers in a survey, and highly positively correlated with both the number of responses in the primary salient answer and the very strong primary salience variable. Therefore we entered each of these variables as alternative indicators of survey difficulty in separate regressions. Similarly, we entered Wright and Extended Wright indexes separately too.

\section{[Tables $7 \mathrm{a}$ and $7 \mathrm{~b}$ about here]}

As expected, survey characteristics crucially affect a family’s matching performance in the feuds. An easier survey -as characterized by a higher coordination index, a stronger overall primary salient answer, and fewer survey entries- makes it more likely that a family will score more points. The effect of later (third and fourth), intentionally easier, feuds is positive and generally significant. We also included dummy variables for survey category (results are not presented), but none had a meaningful effect on the family's performance.

We find that a family's previous playing experience had a significant positive effect on points scored in regressions 1, 2, 4, and 5, but dropped slightly below significant levels in the other two regressions. Based on our estimates, families with experience are able to score approximately two more points than families with no experience. This suggests that experienced families may be more adept at strategically 
manipulating their lineups (position of each family member) to make it more likely that they will cover more survey ground and thus score more points.

We find that race and gender composition do not significantly affect the families' matching performance. Caucasian families comprise nearly $80 \%$ of the sample, thus it comes as no surprise that regressions run with and without the Caucasian dummy variable and for the Caucasian subsample yielded similar results, with the race variable remaining insignificant. Moreover, there are not enough multi-racial groups in our sample to draw any conclusions on the impact of intra-group racial diversity. In terms of gender, including dummy variables for the number of males in the family shows no difference in performance between having one male or more than one male. ${ }^{18}$

On the other hand -and of particular interest to our study- both the generational gap and relatedness indicators have a statistically significant impact on the points that a family is able to score in this game. As we see in Tables 7a and 7b, the Wright Index is negative and statistically significant in all the regressions in Table 7a, while the generational gap is positive and also statistically significant in all of these regressions. ${ }^{19}$ Ceteris paribus, a higher degree of relatedness within a family makes it more likely that they will score fewer points in a feud game, while a wider generational gap makes it more likely that the family will score more points. Based on the conceptual framework we presented, we attribute this to more closely-related and similarly-aged individuals being less able to "think out of the box" when it comes to matching a wide range of

\footnotetext{
${ }^{18}$ These additional ethnicity and gender results are not presented here.

${ }^{19}$ The statistical significance of the Wright index is higher than that of the generational gap across the board.
} 
survey responses. More precisely, individuals who are closely related and/or of similar ages, are more likely to have highly correlated primary and secondary salient labels. Thus they approach this matching problem using more similar sets of information and frames of reference, which results in fewer answers matched and fewer points scored.

Our results also show that a family’s matching performance is strongly driven by the ease of the survey, as all indicators of survey difficulty are highly significant, both in statistical and economic terms. ${ }^{20}$ It appears that all genders and all ethnicities share enough information and frames of reference to successfully match the survey answers that are not too odd or difficult. However, even after controlling for these observable demographics and survey difficulty, we find that more distant relatives (both age and relationship-wise) add dimensions of heterogeneity that are unique enough to significantly increase the chance that a family will match the responses that are typically more odd or difficult. $^{21}$

Our interpretation of the results based on the Wright Index is to some degree reinforced by our results for the Extended Wright Index. As we see in Table 7b, the Extended Wright's coefficient is smaller than that of the Wright and has either a weaker impact or no impact on the number of points scored. ${ }^{22}$ The Extended Wright Index adds a relatedness factor of 0.5 between spouses (the same as siblings and parent/offspring).

\footnotetext{
${ }^{20}$ For instance, a 0.2 increase in the coordination index, would allow a playing family to score about 10 more points in the game.

${ }^{21}$ Hamilton (2005) posits that group heterogeneity is usually associated with the generation and use of unique or non-traditional perspectives and alternatives.

${ }^{22}$ This index modification also lowers the statistical significance of the generational gap.
} 
The weakness and insignificance of the extended index suggests that spouses do not add to the sameness of the group and thus will not hurt the family's matching performance. Presumably, because the spouse grew up in a different household and was exposed to different experiences earlier in life, he/she adds diversity to the playing family. As a corollary, replacing your spouse with an offspring or sibling will most likely cause matching performance to decline.

Observe that making the family even slightly more diverse, either relationship or age-wise, could make the difference between winning and losing a feud. For example, a nuclear family consisting of a husband, wife and their three children has a Wright Index of 4.5. Replacing one child with a son-in-law reduces the Wright Index to 2.5. This two point decline in the index yields approximately 2.5 more points scored for the now more diverse family, when evaluated at the mean coefficient for the Wright index across our three regression models. Going one step further and replacing yet another child with the son-in-law’s sister causes the Wright index to fall to 1.5 and yields roughly 1.2 more points (on top of the 2.5) scored for the family compared to the original nuclear family. These 2 to 4 additional points are precisely the number of points associated with the more difficult-to-match responses in feuds (see Table 5), and can make the difference between winning a feud, and allowing the other family to steal the points you accumulated.

To gain additional insight into the economic significance of the preceding results, we split our sample into "easy" and "difficult" feuds. We define a feud as easy if the coordination index was above the median (0.31697), and difficult if it was below. As seen on Table 8a, when feuds are easy -with the exception of experience- family characteristics do not matter in explaining performance in this game. Since the task is 
relatively simple, the family's success is strongly driven by this simplicity. When feuds are difficult, on the other hand, the significance of the Wright index increases both statistically and economically (see Table 8b). When evaluated at the mean coefficient for the Wright Index across the difficult feud sub-sample in Table 8b, the 2 to 4 point diversity-induced gain we obtained for the entire sample, turns into a 4 to 6 point gain. Thus the positive impact of genetic-based diversity is accentuated when the average difficulty of the task increases.

Now consider the age distribution of the nuclear family used above. Suppose the parents are both 50 years old, while the three children are 22, 20 and 18 years old. By replacing the 20 year-old with a 75 year-old grandparent, we add 5 total generation gaps to the family, which yield 2 additional playing points if we evaluate the outcome using the mean coefficient of total generation gap obtained in the full sample regressions on Table 7a. Making a further swap of a 15 year-old for the 18 year-old adds nearly one more generation to the total gap and brings the additional points the family can earn solidly above 2 when evaluated using the average coefficient for the full sample. At the margin these additional points can make a difference between winning and losing a feud especially one that is more difficult and has more answers to match. A gain of two points falls within one standard deviation of all mean responses greater than the fourth response in a feud (see Table 5).

Based on these results, our advice to Family Feud contestants is to try to assemble as age-diverse and distantly related a group as possible. A grandparent, grand-uncle, inlaw, spouse, or very young second cousin is more likely to help you than a sibling, an offspring, or a parent. 


\section{(ii) Stealing Family Performance}

In the Family Feud, non-playing family members may have the opportunity to steal points from the playing family. A steal attempt occurs when the playing family has accumulated 3 wrong answers in a feud before amassing 300 points. The non-playing family may steal the feud points by correctly matching one of the non-matched answers. These family members are permitted to huddle-up, consult each other, and discuss their one answer before stating it. There were 413 attempts at stealing and 193 successful steals by non-playing families in our sample. Of these, 150 (or 78\%) of the successful steals, were carried out by Caucasian families who also comprise the bulk of our total sample.

The problem of stealing a feud differs from that of playing the feud for two reasons. First, the family members consult each other and come to an agreement on the answer they will give. Unfortunately viewers of the game (and thus researchers) do not hear the discussion and thus have no data on how these discussions are run or who wields influence in them. Second, the likelihood of successfully stealing a feud depends on how well the playing family has so far matched answers to the survey. Although all playing families must get 3 incorrect answers before a steal attempt is possible, the total number of correct answers given by the playing family prior to this point may vary. In an easy feud, the playing family may have correctly matched 5 out of 8 possible feud responses before the steal was possible. On the other hand if the feud is difficult, it is possible that they matched only 2 out of 8 possible responses prior to a steal attempt. The number of survey responses remaining unmatched prior to the steal is thus a measure of the relative 
difficulty of the survey and also takes into account the matching success of the playing family prior to the attempted steal.

In order to analyze the effect of diversity on the stealing family's task, we estimated a logistic regression of the probability of successful stealing. We measure the difficulty of the stealing family's task by the number of survey answers that were not matched by the playing family prior to the steal attempt. Results are shown in Table 9 . The most significant determinant of successful stealing was the number of unmatched answers remaining in the survey. The larger the number of unmatched answers, the more difficult it was for a family to successfully steal the feud. This is consistent with our expectations. The Wright Index was not significant in any of our specifications. This also is not surprising and actually reinforces the result obtained for the unmatched answers. The success of stealing depends mostly on how difficult the survey was and how well the playing family matched survey answers, and not on the relatedness or generational gaps within the non-playing family. Interestingly, the only measure of age diversity that was statistically significant was the maximum age of a player in the stealing family. The older was the oldest family member, the less likely the family was to successfully steal. This suggests that older family members may wield more influence, and perhaps be more resolute, albeit with a more limited frame of reference, during the discussion that precedes giving an answer. Finally, Caucasian families were more likely than other families to successfully steal. Note that $78 \%$ of successful steals were carried out by Caucasian families. Similar to the full sample results, we find no effect of gender on the probability of a successful steal.

\section{Concluding Remarks}


We use a unique data set from the television game show The Family Feud to study group decisions and performance in a naturally occurring experiment. The feud game, which is part of this show, features families competing for monetary prizes by seeking to match the results of public opinion surveys. Because our groups are families, we are able to include relatedness or genetic-based diversity as a measure of group composition in addition to the traditional group heterogeneity measures present in other studies.

Our main result is that controlling for task difficulty, a family's degree of relatedness and the generational distance across its members are the most significant group characteristics explaining performance in this game. Closely related families and families with lower generational variation perform worse than families exhibiting greater diversity in these two dimensions. This indicates that families comprised of members with more diverse backgrounds and ages bring along richer sets of information and frames of reference that allow them to be more proficient at matching the choices of larger subsets of survey respondents. In addition, we find that the economic significance of diversity increases when the average difficulty of the task is higher.

We also explore the issue of the determinants of salience in decisions and games. The subjects responding to the surveys used in the feud game give their choices with no intention of coordinating or matching. As such, these responses are primary salient to this group. Family Feud contestants, on the other hand, seek to match these survey responses, and therefore their choice criterion is different. This criterion (i.e., match what is primary salient to others) is known as secondary salience. Our results indicate that diversity within the playing group positively impacts the accuracy of the group's secondary salient choices. This is consistent with the notion that players' identities and 
personal attributes may matter in simple tasks involving matching and coordination. Thus our paper supports the notion that game and decision theorists should more routinely build models with structures capable of capturing the roles that player personal traits may play in determining strategies and outcomes. Bacharach (1993), and Sugden (1995), among others, make important strides in this direction, but this approach has yet to be incorporated as a general modeling strategy in coordination games or otherwise. We believe that this is an important issue, which may potentially impact the way we think about information, rational decisions, and equilibria even in very simple games and decision problems. 


\section{$\underline{\text { References }}$}

Adamowicz, W., M. Hanemann, J. Swait, R. Johnson, D. Layton, M. Regenwetter, T.

Reimer, and R. Sorkin (2005), “Group Decisions: Analyzing Decision Strategy and

Structure in Households, Mimeo, University of California, Berkeley.

Ancona, D. and D. Caldwell (1992), "Demography and Design: Predictors of New Product Team Performance,” Organization Science 33, 321-341.

Aydinonat, N. E. (2001), “The Theory of Focal Points,” Discussion Papers Series of the Faculty of Political Sciences, Ankara University.

Bacharach, M. (1993), "Variable Universe Games", in Frontiers of Game Theory, ed. K. Binmore, A. Kirkman, and P. Tani, Cambridge, MA, MIT Press. Becker, G. (1973), “A Theory of Marriage: Part I," Journal of Political Economy 81, 813-846.

Egghe, L. (2005), “Zipfian and Lotkaian Continuous Concentration Theory, Journal of the American Society for Information Science and Technology 56, 935-945.

Fleming, R. A., and J. Kaiwi (2002), "The Problem of Unshared Information in Group Decision-Making: A summary of Research and the Implications for Command and Control, SSC-SD Technical Document 3149.

Gauthier, D. (1975), "Coordination,” Dialogue 14, 195-221.

Gruenfeld, D., E. Mannix, K. Williams, and M. Neale (1996), “Group Composition and Decision Making: How Member Familiarity and Information Distribution Affect Process and Performance,” Organizational Behavior and Human Decision Processes 67, 1-15. 
Hamilton, B. H., J. Nickerson, and H. Owan (2003), “Diversity and Productivity in Teams,” Working Paper, John M. Olin School of Business, Washington University in St. Louis.

Hamilton, M. (2004), “The Performance of Difference: Mobilizing Heterogeneous Knowledge, Working Paper, College of Business, University of Rhode Island.

Heath, C., B. Ho, and J. Berger (2006), “Focal Points in Coordinated Divergence,” Journal of Economic Psychology 27, 635-647.

Hinsz, V. B., R. S. Tindale, and D. A. Vollrath (1997), “The Emerging Conceptualization of Groups as Information Processors,” Psychological Bulletin 121, 43-64.

Ijiri, Y., and H. Simon (1977), Skew Distributions and the Size of Business Firms, North Holland.

Janssen, M. (1998), "Focal Points,” The New Palgrave Dictionary of Economics and the Law, ed. P. Newman, 150-155.

Martin, J.A., B. Hamilton, P. Sutton, S. Ventura, F. Menacker, S. Kirmeyer, and T.J. Mathews (2009), “Births: Final Data for 2006,” National Vital Statistics Report. Mehta, J., C. Starmer, and R. Sugden (1994), "The Nature of Salience: An Experimental Investigation of Pure Coordination Games,” American Economic Review 84, 658-673.

Mello, A. and M. Ruckes (2006), “Team Composition,” Journal of Business 79, 10191039.

O’Reilly, C., and S. Flatt (1989), “Executive Team Demography: Organizational Innovation and Firm Performance,” Unpublished Manuscript, University of California at Berkeley. 
Prat, (2002), “Should a Team be Homogeneous?” European Economic Review 46, 11871207.

Schelling, T. (1960), The Strategy of Conflict, Cambridge, MA, Harvard University Press.

Sugden, R. (1995), "A Theory of Focal Points,” Economic Journal 105, 533-550.

Wright, S. (1922), “Coefficients of Inbreeding and Relationship,” American Naturalist 56, 330-338. 


\section{Table 1}

\section{Wright Relatedness Coefficients*}

\begin{tabular}{lc} 
Relationship to Me & \multicolumn{1}{c}{ Relatedness Coefficient } \\
\cline { 2 - 2 } Identical Twin & 0.5 \\
Parent & 0.5 \\
Offspring & 0.5 \\
Sibling & 0.25 \\
Grandparent & 0.25 \\
Grandchild & 0.25 \\
Nephew/Niece & 0.25 \\
Aunt/Uncle & 0.125 \\
Cousin & 0.125 \\
Great-Grandparent & 0.03125 \\
Second Cousin &
\end{tabular}

*The relatedness coefficient can be interpreted as the fraction of genes shared by the two relatives 
Table 2

Playing Family Descriptive Statistics

(524 feuds)

$\underline{\text { Family Characteristics }}$

Experience

Mean Family Age

Head of Family Age

Total Generation Gap

Average Generation Gap

Fraction of Caucasian Families

Male to Female Ratio

Wright Index

Extended Wright Index
Mean

0.4523

35.34

41.52

5.39

0.54

0.784

0.472

3.123

3.305

Race

Caucasian

Black

Hispanic

Asian

Native American

Multiracial
Frequency

411

67

21

16

7

2
1.206

Std. Dev.

9.78

3.05

0.31

0.206

1.291

Percent

78.44

12.79

4.01

3.05

1.34

0.38 
Table 3

\section{Survey Categories}

\begin{tabular}{|c|c|c|}
\hline Feud Category & Frequency & $\underline{\text { Percent }}$ \\
\hline Vocabulary & 137 & 26.15 \\
\hline Show Business & 68 & 12.98 \\
\hline Social & 58 & 11.07 \\
\hline Domestic & 42 & 8.02 \\
\hline Work & 32 & 6.11 \\
\hline History & 31 & 5.92 \\
\hline Parenting & 26 & 4.96 \\
\hline Travel & 24 & 4.58 \\
\hline Food & 23 & 4.39 \\
\hline Finances & 19 & 3.63 \\
\hline Medical & 17 & 3.24 \\
\hline Apparel & 16 & 3.05 \\
\hline Sports & 13 & 2.48 \\
\hline Animals & 9 & 1.72 \\
\hline News & 7 & 1.34 \\
\hline
\end{tabular}


Table 4

\section{Survey Descriptors}

A. Surveys Containing

Very strong primary salience

(> 50\% of responses)

Strong primary salience

(> $40 \%$ of responses)

B. Survey Characteristics

Coordination Index

Total possible

responses in feud

$(\min =3, \max =10)$

C. Mean Characteristics by Feud

\section{Frequency}

265

373

71.18

50.57

$\underline{\text { Percent }}$

$\underline{\text { Mean }}$

Std. Dev.

0.343

0.156

5.22

1.14

Feud Number

1

0.3947

2

3

4

Fraction of surveys

with very strong

primary salience

Coordination Index

0.2058

0.2861

(min=0.0556, $\max =0.8289)$

Average number of

5.74

5.76

answers in survey

Number of responses in

44.8

45.5

primary salient entry

Number of feuds

152
152

152
0.3723

0.5378

4.92

3.56

54.1

68.5

0.8358

0.5789

0.8358

67 
Table 5

\section{Responses and Misses by Rank of Response}

\begin{tabular}{|c|c|c|c|}
\hline Response Rank & $\mathbf{N}$ & $\begin{array}{l}\frac{\text { Mean number }}{\text { of responses }} \\
\underline{\text { (std. dev) }}\end{array}$ & $\frac{\text { Number of times }}{\underline{\text { missed (percent) }}}$ \\
\hline Overall primary salient & 524 & $\begin{array}{l}50.8 \\
(16.8)\end{array}$ & $9(1.72 \%)$ \\
\hline Second & 524 & $\begin{array}{l}19.0 \\
(8.1)\end{array}$ & $51(9.73 \%)$ \\
\hline Third & 523 & $\begin{array}{l}10.1 \\
(5.0)\end{array}$ & $113(21.61 \%)$ \\
\hline Fourth & 491 & $\begin{array}{r}6.1 \\
(3.2)\end{array}$ & $166(33.81 \%)$ \\
\hline Fifth & 410 & $\begin{array}{r}4.4 \\
(2.3)\end{array}$ & $188(45.85 \%)$ \\
\hline Sixth & 162 & $\begin{array}{r}3.9 \\
(1.7)\end{array}$ & $85(52.47 \%)$ \\
\hline Seventh & 54 & $\begin{array}{c}3.0 \\
(0.97)\end{array}$ & $33(61.11 \%)$ \\
\hline Eighth & 17 & $\begin{array}{r}2.8 \\
(1.0)\end{array}$ & $11(64.71 \%)$ \\
\hline Ninth & 7 & $\begin{array}{r}3.4 \\
(2.1)\end{array}$ & $4(57.14 \%)$ \\
\hline
\end{tabular}


Table 6

Feud Descriptive Statistics

Feuds won by

Frequency

$\underline{\text { Percent }}$

Playing Family

330

62.98

Stealing Family

194

37.02

Buzzer Winner

341

65.00

$\underline{\text { Overall primary salient response matched }}$

$\begin{array}{llc}\text { Playing Family } & 507 & 96.76 \\ \text { Stealing Family } & 8 & 2.53 \\ \text { No Family } & 9 & 1.72\end{array}$

Games playing through *

Second Feud

152

29.01

Third Feud

152

29.01

Fourth Feud

67

12.79

* A game consists of all the feuds played by one pair of families in one game 
Table 7a

OLS Regression Results $(\mathrm{N}=524)$

Dependent Variable: Points Earned by Playing Family

(Standard errors in parentheses)

Intercept

Mean Age

Total Gen Gap

Caucasian

Male

Experience

Wright Index

Coordination Index

Number of Primary

Salient Responses

Number of Survey

Answers

Very Strong

Primary Salience

Second Feud

Third Feud

Fourth Feud

Adj. R-squared
1

2

60.0854

63.0893

$(6.0094) * * * *$

$-\mathbf{0 . 1 3 3 6 0}$

(0.1361)

0.4087

$(0.2350) * *$

1.4581

(1.6732)

0.9001

(3.3525)

2.3286

$(1.3897) * *$

$-1.1781$

$(0.5487) * * *$

52.0776

$(5.2448) * * * *$

$-0.1351$

1.440

(1.7081)

0.8666

(3.4220)

2.1923

$-1.2696$
3

82.1383

$(7.8261)^{* * * *}$

$-0.1086$

(0.1428)

(0.1390)

0.4024

(0.2456)*

$(0.2402)$ *

1.3228

(1.7455)

0.9535

(3.5013)

1.7782

$(1.4183)^{*}$

(1.4449)

$-1.3927$

$(0.5597) * * *$

$(0.5703) * * *$
0.4089

$(0.0475) * * * *$

$-1.4075$

$(0.8225) * *$

10.1809

$(1.5334) * * * *$

$$
\begin{array}{r}
-1.8943 \\
(1.7931)
\end{array}
$$

$-2.0726$

$-1.7797$

(1.8303)

(1.8673)

\subsection{4}

(1.8479)

3.1082

$(1.8791) * *$

3.8553

$(1.9833) * *$

3.807

(2.6508)*

7.3134

$(2.5988) * * * *$

9.3738

$(2.9840) * * * *$
**** denotes significance at the $1 \%$ level

*** denotes significance at the 5\% level
** denotes significance at the $10 \%$ level

* denotes significance at the 15\% level 
Table 7b

OLS Regression Results $(\mathrm{N}=\mathbf{5 2 4})$

Dependent Variable: Points Earned by Playing Family

(Standard errors in parentheses)

4

Intercept

Mean Age

Total Gen Gap

Caucasian

Male

Experience

Extended Wright

Coordination Index

Number of Primary

Salient Responses

Number of Survey

Answers

Very Strong

Primary Salience

Second Feud

Third Feud

Fourth Feud

Adj. R-squared
61.3529

$(6.0165) * * * *$

$-0.1108$

(0.1357)

0.3729

$(0.2358) *$

1.5245

(1.6771)

0.6685

(3.3732)

2.4784

$(1.3904) * *$

$-\mathbf{0 . 8 3 4 6}$

(0.5875)

52.6429

$(5.2462) * * * *$
5

58.0137

$(6.3758) * * * *$

$-0.1097$

(0.1387)

0.3498

(0.2412)*

1.5126

(1.7129)

0.6400

(3.4448)

2.3548

$(1.4198) * *$

$-0.8544$

(0.6001)

$(0.6117) * *$
80.0728

$(7.7886) * * * *$

$-0.0814$

(0.1423)

0.3637

$(0.2463)$ *

1.3889

(1.7502)

0.6748

(3.5234)

1.9438

(1.4465)

$-1.0474$ 
Table 8a

OLS Regression Results

Dependent Variable Points Earned by Playing Family

High Coordination Index $(>\mathbf{0 . 3 1 6 9 7})$ Sub-sample $(\mathrm{N}=\mathbf{2 6 2})$

1

78.7555

$(6.3469) * * * *$

$-0.06480$

(0.1408)

0.1431

(0.2220)

0.4760

(1.6827)

2.5521

(3.2830)

$-2.6598$

$(1.3892) * *$

$\mathbf{- 0 . 2 7 8 2}$

(0.5347)

12.0987

Coordination

$(6.3046) * *$
2

82.9883

$(6.9902) * * * *$

$-0.0560$

(0.1418)

0.1402

(0.2237)

0.3630

(1.6939)

2.8344

(3.3053)

$-2.8546$

$(1.3979) * * *$

$-\mathbf{0 . 3 0 6 0}$

(0.5384)

0.0122

(0.0655)

Number of Primary

Salient Reponses

Primary Salience
Number of Survey

Answers

Very Strong

Second Feud

0.9549

(2.1252)

Third Feud

4.5212

$(1.8698) * * *$

Fourth Feud

8.3140
$(2.1826) * * * *$

.1117

$-1.7264$

$(0.9467) * *$

$-6.2156$

$(2.5450) * * *$

0.9776

1.6695

(2.1407)

(2.1266)

4.8643

3.4071

$(1.8756) * * *$

$(1.9330) * *$

9.7629

6.2713

$(2.1144) * * * *$

$(2.6299) * * *$

Adjusted R-square

.0988

.1296

a/ Results using the extended Wright Index were similar in terms of both magnitude and statistical significance of all the variables.

**** denotes significance at the $1 \%$ level

*** denotes significance at the $5 \%$ level
** denotes significance at the $10 \%$ level

*denotes significance at the $15 \%$ level 
Table $8 b$

OLS Regression Results

Dependent Variable: Points Earned by Playing Family

Low Coordination Index $(\leq \mathbf{0 . 3 1 6 9 7})$ Sub-sample $(\mathrm{N}=\mathbf{2 6 2})$

1

Intercept

Mean Age

Total Generation

Gap

Caucasian

Male

Experience

Wright Index ${ }^{\mathrm{a} /}$

Coordination

Index

Number of Primary

Salient Reponses

Number of Survey

Answers

Very Strong

Primary Salience

Second Feud

$-3.2071$

(2.6050)

$-1.4027$

(3.0901)

$-2.5761$

(8.6137)

.1435

Third Feud

Fourth Feud

111.3715

Adjusted R-square
2

56.0256
$(10.9047) * * * *$

$-0.0601$

(0.2292)

0.5669

(0.4301)

1.3233

(2.8498)

0.7109

(5.9119)

6.7064

$(2.4065) * * * *$

$-2.1307$

$(0.9700) * * *$
$-2.3634$

$(0.9895) * * *$

3

74.2929

$(12.7040) * * * *$

$-0.0563$

(0.2352)

0.7090

(0.4379)*

1.0021

(2.9042)

0.8599

(6.0486)

6.5289

$(2.4612)^{* * * *}$$$
(0.9895)^{* * *}
$$

0.4341

$(0.1411)^{* * * *}$

$(19.9005) * * * *$

$-3.4510$

(2.7123)

$-0.4533$

(1.2269)

2.4026

(4.3196)

$-3.3231$

(2.7709)

$-0.1477$

1.0894

(3.2128)

(3.3625)

$\overline{a / \text { Results using the extended Wright Index were similar in terms of both magnitude and statistical }}$ significance of all the variables.

**** denotes significance at the $1 \%$ level

3.1663

5.5409

(8.8748)

(9.2724)

*** denotes significance at the $5 \%$ level

** denotes significance at the $10 \%$ level *denotes significance at the $15 \%$ level 
Table 9

Logistic Regression Results ( $\mathrm{N}=413$ attempted steals) Dependent Variable: Probability of Successful Steal (Standard errors in parentheses)

\begin{tabular}{|c|c|c|c|}
\hline & 1 & 2 & 3 \\
\hline Intercept & $\begin{array}{c}1.1203 \\
(0.9013)\end{array}$ & $\begin{array}{l}1.3095 \\
(0.7409) * *\end{array}$ & $\begin{array}{c}0.7374 \\
(0.6260)\end{array}$ \\
\hline Mean Age & $\begin{array}{l}-0.0229 \\
(0.0216)\end{array}$ & & \\
\hline Maximum Age & & $\begin{array}{l}-0.0303 \\
(0.0166) * *\end{array}$ & \\
\hline Head Age & & & $\begin{array}{l}-0.0132 \\
(0.0119)\end{array}$ \\
\hline Total Generation Gap & $\begin{array}{l}-0.0048 \\
(0.0371)\end{array}$ & $\begin{array}{c}0.0575 \\
(0.0543)\end{array}$ & $\begin{array}{c}0.0022 \\
(0.0394)\end{array}$ \\
\hline Caucasian & $\begin{array}{c}0.3813 \\
(0.2401)^{*}\end{array}$ & $\begin{array}{c}0.3967 \\
(0.2404) * *\end{array}$ & $\begin{array}{l}0.3746 \\
(0.2393)^{*}\end{array}$ \\
\hline Male & $\begin{array}{c}0.4475 \\
(0.5274)\end{array}$ & $\begin{array}{c}0.5269 \\
(0.5244)\end{array}$ & $\begin{array}{c}0.5472 \\
(0.5255)\end{array}$ \\
\hline Experience & $\begin{array}{l}-0.1681 \\
(0.2101)\end{array}$ & $\begin{array}{l}-0.1308 \\
(0.2118)\end{array}$ & $\begin{array}{l}-0.1699 \\
(0.2101)\end{array}$ \\
\hline Wright Index & $\begin{array}{c}0.0254 \\
(0.0888)\end{array}$ & $\begin{array}{c}0.0274 \\
(0.0880)\end{array}$ & $\begin{array}{c}0.0483 \\
(0.0875)\end{array}$ \\
\hline Unmatched Answers & $\begin{array}{l}-0.5883 \\
(0.1084) * * * *\end{array}$ & $\begin{array}{l}-0.5895 \\
(0.1088) * * * *\end{array}$ & $\begin{array}{l}-0.5918 \\
(0.1091)^{* * * * *}\end{array}$ \\
\hline Second feud & $\begin{array}{c}0.1842 \\
(0.2609)\end{array}$ & $\begin{array}{c}0.1905 \\
(0.2614)\end{array}$ & $\begin{array}{c}0.1611 \\
(0.2610)\end{array}$ \\
\hline Third feud & $\begin{array}{l}-0.1062 \\
(0.2749)\end{array}$ & $\begin{array}{l}-0.0882 \\
(0.2760)\end{array}$ & $\begin{array}{c}-0.1172 \\
(0.2748)\end{array}$ \\
\hline Fourth feud & $\begin{array}{l}-0.2389 \\
(0.4110)\end{array}$ & $\begin{array}{l}-0.2237 \\
(0.4105)\end{array}$ & $\begin{array}{c}-0.3084 \\
(0.4070)\end{array}$ \\
\hline -2 Log Likelihood & 531.82 & 529.58 & 531.70 \\
\hline Likelihood Ratio & $38.9541 * * * *$ & $41.1951 * * * *$ & $39.0714 * * * *$ \\
\hline
\end{tabular}

**** denotes significance at the $1 \%$ level *** denotes significance at the 5\% level
** denotes significance at the $10 \%$ level *denotes significance at the $15 \%$ level 